\title{
Mid-Term Clinical Outcomes of Intraarticular Injection of Platelet Rich Plasma and Adipose-Derived Mesenchymal Stem Cells for the Treatment of Intra articular Pathology of the Knee, Hip and Shoulder
}

\section{Steele JR, Boggess BR*, Finley S and Reinke E \\ Department of Orthopaedic Surgery, Duke University Medical Center, USA}

*Corresponding author: Blake Boggess, Department of Orthopaedic Surgery, Duke University Medical Center, Box 3000, 40 Medicine Circle, Room 5309, Durham, NC 27710, USA, Tel: (919) 684-3170; Email: blake.boggess@duke.edu

\section{Research Article \\ Volume 3 Issue 2}

Received Date: October 11, 2019

Published Date: October 22, 2019

DOI: $10.23880 /$ jes- 16000130

\section{Abstract}

Introduction: Intra articular injections of platelet rich plasma (PRP) and adipose-derived mesenchymal stem cells (ADMSCs) have individually shown potential benefit in the treatment of intraarticular pathology of the knee, hip and shoulder. However, outcomes data is sparse in regards to PRP and AD-MSCs injected in the same setting. The purpose of this study is to assess the midterm clinical outcomes and safety profile of intraarticular injection of PRP and AD-MSCs into arthritic hips, knees and shoulders.

Methods: After IRB approval, a retrospective chart review identified 50 patients who had received intraarticular injection of PRP and AD-MSCs into their hip, knee or shoulder for intraarticular pathology. Clinical outcomes including the need for further injections, progression to surgery and any adverse effects were collected. Patient reported outcomes scores and patient satisfaction were collected at average 2-year follow-up for 39/50 patients (78\%).

Results: Clinical outcomes were favorable at 2-year follow-up. Approximately $70 \%$ of people were satisfied with the results of their treatment overall. Only $4 \%$ of patients progressed to repeat PRP and AD-MSC injection into the joint of interest while $21 \%$ of patients progressed to surgery. There were no significant adverse events encountered during the 2 year period. Median improvement in clinical outcome scores was above the minimally clinically important difference.

Conclusion: Intraarticular injections of PRP and AD-MSCs into hips, knees and shoulders are safe and have a low rate of progression to further surgery or repeat injection at 2-year follow-up. Improvement in clinical outcome scores and high patient satisfaction were demonstrated at 2-year follow-up.

Keywords: Platelet Rich Plasma; Intraarticular Injections; Anti-Inflammatory Functions; Pathology 


\section{Journal of Embryology \& Stem Cell Research}

\section{Introduction}

Musculoskeletal disorders are among the most prevalent chronic conditions and a leading cause of global disability and pain [1,2]. Osteoarthritis and degenerative intraarticular soft tissue injuries such as rotator cuff and meniscal tears make up a large proportion of these disorders, causing significant morbidity and economic burden. As such, it is imperative to find effective treatments for these intraarticular disorders. Currently, non-operative treatments such as lifestyle modification, NSAIDs, intraarticular corticosteroid injections and physical therapy seek to mitigate the symptoms of these diseases but do not necessarily halt progression. Surgery may be warranted if symptoms become severe. Intraarticular injection of orthobiologics including platelet rich plasma (PRP) and mesenchymal stem cells (MSCs) represent another treatment option that can aim to both improve symptoms associated with intraarticular pathologies as well as promote regeneration of damaged tissues. PRP contains many growth factors that have important roles in wound healing and tissue regeneration. Intraarticular PRP injection has been shown to be helpful in cartilage regeneration and attenuates arthritis in animal models $[3,4]$.

Research has shown that MSCs have the ability to differentiate into osteocytes, adipocytes, myocytes and chondrocytes [5-7]. In addition, MSCs possess powerful immunosuppressive and anti-inflammatory functions in the intraarticular environment [6,7]. Autologous stem cells can be derived from bone marrow, adipose tissue and the umbilical cord, among other sources. In the current regulatory environment only minimally manipulated MSCs can be administered at the time of acquisition, making stem cells acquired from bone marrow and adipose tissue the most commonly used [8]. Studies have shown similar chondrogenic potential between adipose-derived and bone marrow-derived MSCs [9].

Multiple animal and human studies have been performed in order to research the role of autologous MSCs in the treatment of osteoarthritis and intraarticular soft tissue defects. The vast majority of AD-MSCs research has centered around the role of in the treatment of knee osteoarthritis. Several studies have shown a favorable safety profile and good clinical outcomes at short to medium term follow-up, with improvements in pain and patient-reported outcome scores [10-15]. The majority of these injections were performed in a surgical setting rather than the office setting. There have been a smaller number of studies researching the role of autologous MSCs in the treatment of hip or shoulder pathology. In regards to shoulder, the majority of research has surrounded the use of autologous MSCs for treatment of rotator cuff pathology rather than glenohumeral arthritis. One study showed that autologous MSCs may help improve re-tear rates used as an adjunct along with rotator cuff repair [16]. Another study showed improvements in pain and function as well as evidence of tendon regeneration when autologous MSCs were used as a standalone treatment of rotator cuff tears [17]. Autologous MSCs have also been shown to be effective in reducing pain and improving function in patients with hip osteoarthritis $[18,19]$.

Van Pham et al showed that PRP with MSCs improved healing of injured cartilage in a murine model compared to MSCs alone [20]. This study demonstrated that PRP promoted MSCs proliferation and differentiation into chondrogenic cells and increased expression of collagen 11, Sox 9 and aggrecan. Also, PRP inhibited expression of the angiogenic factor vascular endothelial growth factor [20].

As detailed above, the majority of clinical research surrounding autologous MSCs has looked at their effectiveness in the treatment knee osteoarthritis and has been performed in the surgical setting. Our study is unique in that both PRP and MSCs were injected in the same setting and for a variety of joints and pathologies. In this study, intraarticular injections of PRP and AD-MSCs were performed in the office into the shoulder, hip and knee joints. Pathology included osteoarthritis, rotator cuff tears, tendinitis, meniscus tears and labral tears. The aim of the study was to evaluate the safety profile and patientreported outcomes of office-based intraarticular injections of PRP and AD-MSCs into the shoulder, hip and knee.

\section{Methods}

This is a retrospective cohort study of platelet rich plasma and mesenchymal stem cells delivered by intraarticular injection into the knee, hip and shoulder. Fifty patients between the ages of 18 and 81 who received a PRP injection and AD-MSC injection into the knee, hip or shoulder for intraarticular pathology were included in the study. Intraarticular pathology included osteoarthritis, tendon tears, tendinitis, meniscal tears and labral tears. Only patients with at least one year follow up were included in the study. The data that support the findings of this study are available on request from the 


\section{Journal of Embryology \& Stem Cell Research}

corresponding author. The data are not publicly available due to privacy or ethical restrictions. Study data were collected and managed using REDCap electronic data capture tools hosted at Duke University [21].

Injections were performed by one physician who has done over 20,000 ultrasound-guided intraarticular injections and was trained by plastic surgeons to perform simple lipoaspirations. The Harvest AdiPrep Adipose Concentration System and the Harvest Platelet Concentration System (Terumo BCT, Lakewood, CO) were used. After the risks and benefits of the procedure were explained, written consent was given, and time-out was performed. First, the PRP harvest was performed by drawing 60 cubic centimeters of blood from the antecubital fossa. This was spun in the Harvest centrifuge for 14 minutes to obtain 8-10cubic centimeters of platelet rich plasma.

The patient was then prepped and carefully positioned on the table. An area on the patient's flank and buttocks was then prepped with and surgical prep solution of chlorhexidine and was anesthetized with 5 milliliters of $1 \%$ lidocaine using the 25 gauge $1-1 / 2$ inch needle. An \#11 blade was then introduced to create a track down to the fat graft. The tumescence solution (mixture of 500 cubic centimeters of normal saline, 50 cubic centimeters of $2 \%$ lidocaine with 1 cubic centimeter of epinephrine 1:1000) was injected into the area for fat grafting. This area was then allowed to rest for 12 minutes. Then approximately 30 cubic centimeters of fat graft was removed through a cannula on a 30cc syringe applying suction. The $30 \mathrm{cc}$ of lipoaspirate was then placed in the harvest centrifuge spun for 4 minutes. The knee, shoulder or hip was then injected with the harvested PRP and lipoaspiration of MSCs under ultrasound guidance. Routine orthopedic post-injection instructions were given.

Patient-reported outcomes were obtained over the phone at least one year following injection and at an average of nearly two years. Data collected include patient satisfaction, VAS pain, HOOS Jr for patients with hip pathology, KOOS Jr for patients with knee pathology and ASES for patients with shoulder pathology. Patients were also asked about any side effects from the injections; whether they had any further injections into the joint of interest and whether they went on to have surgery on the joint of interest. Need for further injections were also corroborated through chart review so as to not miss any repeat injections due to recall bias.

\section{Results}

The mean age of the patients in this study was 54 . Thirty patients received the intraarticular injection into the knee, 12 patients received the injection into the shoulder and 8 received the injection into the hip. Table 1 includes demographic information for the cohort broken down by the joint injected. Complete outcomes data was obtained in $78 \%$ of the entire cohort, with $88 \%$ of the hip patients having complete follow-up, $83 \%$ of knee patients, and $58 \%$ of shoulder patients. Median time to final followup was 692 days for the cohort.

\begin{tabular}{|c|c|c|c|c|}
\hline Demographic Information & Hip & Knee & Shoulder & All joints \\
\hline Number of patients & 8 & 30 & 12 & 50 \\
\hline Patients with complete follow-up & $7(88 \%)$ & $25(83 \%)$ & $9(75 \%)$ & $41(82 \%)$ \\
\hline Median follow-up (years) & 1.9 & 2 & 1.7 & 1.9 \\
\hline
\end{tabular}

Table 1: Demographic info broken down by joint injected.

\section{Clinical Outcomes}

The PRP and AD-MSC injections in the same setting were found to be safe, with no severe adverse events and only low rates of minor adverse events. There were no deaths and no severe adverse events including infection or numbness encountered during the study period. Only minor adverse events were reported at an overall rate of $26 \%$. These included 1 skin reaction (2\%), 3 bruises (6\%) and 9 instances of transient swelling (18\%). Less than half of the patients $(46 \%)$ experienced any pain from the injection.
Similarly, the rates of repeat injection or progression to surgery were low in this study. $31 \%$ of patients had an additional injection into the joint of interest during the study period, with the majority of these being corticosteroid injections (27\%) and only $4 \%$ of patients having repeat PRP and AD-MSC injection. $21 \%$ of patients progressed to surgery on the joint which had received the PRP and AD-MSC intraarticular injection. Surgery was joint replacement in $50 \%$ of the overall cohort, however there were differences depending on the joint involved. $100 \%$ of surgeries on the hip $(1 / 1)$ or shoulder $(2 / 2)$ 


\section{Journal of Embryology \& Stem Cell Research}

were joint replacement, while only $20 \%(1 / 5)$ of knee surgeries were joint replacement.

\section{Patient-Reported Outcomes}

Patient-reported outcomes were favorable at 2-year follow-up (Table 2). VAS pain scores improved from a median of 7.0 before injection to 2.5 at final follow-up. VAS pain scores improved an average of 6 points for the knee, 5 points for the hip and 2 points for the shoulder. Median outcome scores as measured by HOOS Jr, KOOS Jr and ASES improved from 51.2 before injection to 76.3 at final follow-up. Similar improvements were seen in the knee (52.5 to 76.3) and hip (57.3 to 76.8), with larger improvements seen in the shoulder (36.7 to 71.7). Of note, patients reported either the same or increased activity at final follow-up compared to activity levels before injection, indicating that improvements in pain and functional outcome scores were not secondary to decreased activity.

\begin{tabular}{|c|c|c|c|c|}
\hline Patient-reported Outcome & Hip & Knee & Shoulder & All joints \\
\hline Patient Satisfaction & $71 \%$ & $68 \%$ & $66 \%$ & $68 \%$ \\
\hline Pre-injection VAS pain & 8 & 8 & 7 & 7 \\
\hline Post-injection VAS pain & 3 & 2 & 4 & 2.5 \\
\hline Pre-injection outcome score (HOOS Jr, KOOS Jr, ASES) & 57.3 & 52.5 & 36.7 & 51.2 \\
\hline Post-injection outcome score (HOOS Jr, KOOS Jr, ASES) & 76.8 & 76.3 & 71.7 & 76.3 \\
\hline
\end{tabular}

Table 2: Patient-Reported Outcomes at 2-year follow-up.

Overall, $69 \%$ of patients were satisfied with the results of their treatment, with 38\% being very satisfied and 31\% being somewhat satisfied. Rates of overall satisfaction were similar between the knee, hip and shoulder joints. $68 \%$ of patients were satisfied with the treatment's ability to improve their pain, with $51 \%$ of patients being very satisfied. $68 \%$ of patients were satisfied with the results of the treatment in improving their ability to do home or yard work, while $69 \%$ of patients reported satisfaction with their improvement in ability to do recreational activities.

\section{Discussion}

This study reports that intraarticular injection of PRP and AD-MSCs into hips, knees and shoulders for intraarticular pathology is a safe treatment that can be performed in the office. The procedure is associated with low rates of progression to surgery and improvement in patient-reported outcomes in medium-term follow-up. In this study, there were no severe adverse reactions to the injection and only a $26 \%$ rate of minor adverse effects including transient swelling, bruising and skin reaction. There were also low rates of additional injection (31\%) and progression to surgery (21\%). $69 \%$ of patients were satisfied with the results of their treatment, and there were improvements in VAS pain scores as well as patientreported outcome scores for all joints involved.

This study is unique in that PRP and adipose-derived MSCs were injected in the same setting. These orthobiologics are often injected separately, likely since injecting them simultaneously takes more time, costs more money for kits and the FDA regulates that they may only be minimally manipulated. Animal models have shown that injecting PRP and AD-MSCs together can have synergistic beneficial effects. This is one of the first studies showing that injecting them simultaneously in humans can be safe and effective in treating osteoarthritis and other soft tissue pathology of the shoulder, hip and knee.

There are some limitations to this study. First, patientreported outcomes were obtained retrospectively which introduces recall bias. Recall bias could potentially impact the change in outcome scores before and after the injection. However, even if this were true it is important to note that patients did feel as if their symptoms and function improved after the injection. Second, the patient population was heterogenous and there were no controls included in the study. This makes it difficult to draw scientific conclusions on the effectiveness of PRP and ADMSCs because they were used to treat several entities. However, it was our goal to evaluate the use of PRP and AD-MSCs in the same clinic setting and it is our belief that this research demonstrates that this treatment is safe, results in a low progression to surgery or repeat injection in the medium-term, and that patients believe that it helps their symptoms. This is valuable information for practitioners running a clinical practice. A larger randomized controlled trial is necessary to further evaluate the effect of the combination of PRP and ADMSCs as a treatment for various intraarticular pathologies 


\section{Journal of Embryology \& Stem Cell Research}

of the shoulder, hip and knee. Last, there were no objective measures such as structural evaluations via ultrasound or MRI. This objective data would be useful in order to correlate any potential objective changes with patients' subjective improvements.

In summary, we demonstrated that intraarticular injection of PRP and AD-MSCs can be performed in the same office setting into the shoulder, hip and knee joints for a variety of pathologies and with a favorable safety profile. The treatment resulted in high satisfaction rates, low rates of progression to repeat injection or surgery, and improvement in patient-reported outcomes over a 2year period. Further studies are needed in order to fully elucidate the role of the combination of PRP and AD-MSCs in the treatment of intraarticular pathology.

\section{Declarations}

Ethics approval was obtained by the Duke University Hospital System IRB prior to the research study beginning. As this was a retrospective study, consent to participate was waived. Consent for publication is not applicable for this study. The data that support the findings of this study are available on request from the corresponding author. The data are not publicly available due to privacy or ethical restrictions. The authors declare that they have no competing interests. There was no funding provided for this study. JS performed conceptualization, data curation, formal analysis and writing. BB performed conceptualization, data curation, formal analysis, writing and supervision. SF performed conceptualization and data curation. ER performed conceptualization, data curation, formal analysis, writing and supervision. The authors sincerely thank Alexander B Oldweiler, BS; Kwadwo A Owusu-Akyaw, MD; and C T Moorman III, MD for their contributions to this project.

\section{References}

1. Brooks PM (2006) The burden of musculoskeletal disease-a global perspective. Clin rheumatol 25(6): 778-781.

2. Lawrence RC, Helmick CG, Arnett FC, Deyo RA, Felson Dt, et al. (1998) Estimates of the prevalence of arthritis and selected musculoskeletal disorders in the United States. Arthritis and rheumatism 41(5): 778-799.

3. Kwon DR, Park GY, Lee SU (2012) The effects of intraarticular platelet-rich plasma injection according to the severity of collagenase-induced knee osteoarthritis in a rabbit model. Ann Rehabil Med 36(4): 458-465.

4. Lippross S, Moeller B, Haas H, Tohidnezhad M, Steubesand N, et al. (2011) Traarticular injection of platelet-rich plasma reduces inflammation in a pig model of rheumatoid arthritis of the knee joint. Arthritis Rheum 63(11): 3344-3353.

5. Friedenstein AJ, Piatetzky S II, Petrakova KV (1966) Osteogenesis in transplants of bone marrow cells. J Embryol Exp Morphol 16(3): 381-390.

6. Chen FH, Tuan RS (2008) Mesenchymal stem cells in arthritic diseases. Arthritis Res Ther 10(5): 223.

7. Barry F, Murphy M (2013) Mesenchymal stem cells in joint disease and repair. Nature reviews Rheumatology 9(10): 584-594.

8. Carballo CB, Lebaschi A, Rodeo SA (2017) Cell-based approaches for augmentation of tendon repair. Techniques in shoulder \& elbow surgery 18(3): e6e14.

9. Xu L, Liu Y, Sun Y, Wang B, Xiong Y, et al. (2017) Tissue source determines the differentiation potentials of mesenchymal stem cells: a comparative study of human mesenchymal stem cells from bone marrow and adipose tissue. Stem Cell Res Ther 8(1): 275.

10. Emadedin $\mathrm{M}$, Labibzadeh $\mathrm{N}$, Liastani MG, Karimi A, Jaroughi N, et al. (2018) Intra-articular implantation of autologous bone marrow-derived mesenchymal stromal cells to treat knee osteoarthritis: a randomized, triple-blind, placebo-controlled phase 1/2 clinical trial. Cytotherapy 20(10): 1238-1246.

11. Roato I, Belisario DC, Compagno M, Lena A, Bistolfi A, et al. (2019) Concentrated adipose tissue infusion for the treatment of knee osteoarthritis: clinical and histological observations. Int Orthop 43(1): 15-23.

12. Jo CH, Chai JW, Jeong EC, Oh S, Shin JS, et al. (2017) Intra-articular Injection of Mesenchymal Stem Cells for the Treatment of Osteoarthritis of the Knee: A 2Year Follow-up Study. The American journal of sports medicine 45(12): 2774-2783.

13. Jo CH, Lee YG, Shin WH, Kim H, Chai JW, et al. (2014) Intra-articular injection of mesenchymal stem cells 


\section{Journal of Embryology \& Stem Cell Research}

for the treatment of osteoarthritis of the knee: a proof-of-concept clinical trial. Stem cells 32(5): 12541266.

14. Lamo Espinosa JM, Mora G, Blanco JF, Granero-Moltó F, Nuñez Córdoba JM, et al. (2016) Intra-articular injection of two different doses of autologous bone marrow mesenchymal stem cells versus hyaluronic acid in the treatment of knee osteoarthritis: multicenter randomized controlled clinical trial (phase I/II). J Transl Med 14(1): 246.

15. Koh YG, Choi YJ, Kwon SK, Kim YS, Yeo JE (2015) Clinical results and second-look arthroscopic findings after treatment with adipose-derived stem cells for knee osteoarthritis. Knee Surg Sports Traumatol Arthrosc 23(5): 1308-1316.

16. Kim YS, Sung CH, Chung SH, Kwak SJ, Koh YG (2017) Does an Injection of Adipose-Derived Mesenchymal Stem Cells Loaded in Fibrin Glue Influence Rotator Cuff Repair Outcomes? A Clinical and Magnetic Resonance Imaging Study. Am J Sports Med 45(9): 2010-2018.

17. Jo CH, Chai JW, Jeong EC, Oh S, Kim PS, et al. (2018) Intratendinous Injection of Autologous Adipose
Tissue-Derived Mesenchymal Stem Cells for the Treatment of Rotator Cuff Disease: A First-In-Human Trial. Stem cells 36(9): 1441-1450.

18. Darrow M, Shaw B, Darrow B, Wisz S (2018) ShortTerm Outcomes of Treatment of Hip Osteoarthritis with 4 Bone Marrow Concentrate Injections: A Case Series. Clin Med Insights Case Rep 11:1179547618791574.

19. Mardones R, Jofre CM, Tobar L, Minguell JJ (2017) Mesenchymal stem cell therapy in the treatment of hip osteoarthritis. J Hip Preserv Surg 4(2): 159-163.

20. Van Pham P, Bui KH, Ngo DQ, Vu NB, Truong NH, et al. (2013) Activated platelet-rich plasma improves adipose-derived stem cell transplantation efficiency in injured articular cartilage. Stem Cell Res Ther 4(4): 91.

21. Harris PA, Taylor R, Thielke R, Payne J, Gonzalez N, Conde JG (2009) Research electronic data capture (REDCap)-a metadata-driven methodology and workflow process for providing translational research informatics support. J Biomed Inform 42(2): 377-381. 